\title{
Collaborative Signal and Information Processing for Target Detection with Heterogeneous Sensor Networks
}

\section{Minghui $\mathrm{Li}^{1 *}$, Yilong $\mathrm{Lu}^{2}$ and $\mathrm{Bo} \mathrm{He}^{3}$}

${ }^{1}$ School of Engineering, University of Glasgow, Glasgow, United Kingdom

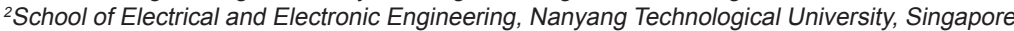

${ }^{3}$ School of Information Science and Engineering, Ocean University of China, Qingdao, China

\begin{abstract}
In this paper, an approach for target detection and acquisition with heterogeneous sensor networks through strategic resource allocation and coordination is presented. Based on sensor management and collaborative signal and information processing, low-capacity low-cost sensors are strategically deployed to guide and cue scarce highperformance sensors in the network to improve the data quality, with which the mission is eventually completed more efficiently with lower cost. We focus on the problem of designing such a network system in which issues of resource selection and allocation, system behavior and capacity, target behavior and patterns, the environment, and multiple constraints such as the cost must be addressed simultaneously. Simulation results offer significant insight into sensor selection and network operation, and demonstrate the great benefits introduced by guided search in an application of hunting down and capturing hostile vehicles on the battlefield.
\end{abstract}

Keywords: Sensor management; Collaborative signal and information processing; Heterogeneous sensor networks; Array signal processing

\section{Introduction}

With the advancements in technologies, it is becoming increasingly feasible to conceive and deploy large-scale sensor networks for a wide variety of applications such as elderly assistance, traffic control, homeland security, military surveillance, and environmental monitoring [1]. Significant work has gone into the development of algorithms to perform a variety of tasks including detection, localization, classification, identification and tracking of one or more targets in the sensor field, and numerous approaches based on Collaborative Signal and Information Processing (CSIP) have been proposed in the literature, see, for example [2,3]. In the scenarios involving multiple targets, data association of measurements from multiple sensors is known to be NP-hard. Multiple hypothesis tracking (MHT) [4] and Markov chain Monte Carlo data association (MCMCDA) [5] are possible solutions to this tough problem at the cost of long latency and extensive computation.

Processing the signal from distributed but networked sensors collaboratively can be treated as an extension of multi-channel array signal processing, where the multiple sensors are collocated with a typical spacing of half a wavelength of the impinging waves and the centralized algorithms based on the relative phases and amplitudes of the wave across the sensors are utilized to obtain the optimal solutions. Array signal processing is essential in most sensor array based systems and has made significant success in a wide variety of applications including modern radar [6-12], underwater sonar [13-15], wireless communications [16-21], intelligent transportation systems [22-24], non-destructive evaluation [25-27], and ultrasound imaging [28]. However, the applications of these algorithms to wireless sensor networks are not straightforward, mainly due to the constraints intrinsic to most of the sensor nodes on energy, communications, computation and size, and the distributed, simple and efficient alternatives that can deliver sub-optimal solutions while meeting the practical constraints have received considerable attention.

We address the problem of target detection and acquisition with a heterogeneous sensor network in this paper. We take as a sample task the problem of hunting down and capturing hostile vehicles on the battlefield, with a sensor network consisting of Unattended Ground Sensors (UGS) and Unmanned Aerial Vehicles (UAV). A UGS may be one sensor node equipped with collocated multi-modality sensors or a small cluster of distributed sensors, but assume to be simple in function, cheap, static but rapidly deployable. The location of each UGS is assumed to be known in advance obtained from on-board GPS receivers or a self-localization algorithm. A UAV is very expensive, mobile and with high performance, equipped with cameras and the necessary image recognition software, and would be able to identify and localize the potential targets with high accuracy. The goal of the whole network system is to distinguish the hostile vehicles from the civilian vehicles, and then to locate and track the hostile vehicles, and eventually capture or destroy the hostile vehicles. There are many real-world applications with parallel environment and requirements such as search and rescue options, surveillance, tracking of moving parts, and search and capture missions. In some cases, the potential targets are intelligent and actively avoiding being captured as in the application addressed in this paper, whereas in other cases their motion is approximately random as in rescue options.

The problem is to study how to guide the UAVs effectively with a network of UGS nodes, and then capture the targets efficiently and accurately. A key element of this problem is the assumption that resources are limited, for example, constrained by the budget, and the choices are driven by trade-offs between the performance and the cost. The capability of the above-mentioned network to perform its tasks depends on several factors, including the amount of available UGS/

*Corresponding author: Minghui Li, School of Engineering, University of Glasgow, Glasgow, United Kingdom, Tel: +44-141-548-2463, E-mail: minghui.li@ieee.org

Received September 19, 2013; Accepted November 14, 2013; Published November 25, 2013

Citation: Li M, Lu Y, He B (2013) Collaborative Signal and Information Processing for Target Detection with Heterogeneous Sensor Networks. Sensor Netw Data Commun 1: 112. doi: 10.4172/2090-4886.1000112

Copyright: ( $2013 \mathrm{Li} \mathrm{M}$, et al. This is an open-access article distributed under the terms of the Creative Commons Attribution License, which permits unrestricted use, distribution, and reproduction in any medium, provided the original author and source are credited. 
UAV sensors, the sensory range and accuracy, the sensor deployment strategy and lifetime, environment characteristics, target behaviors, and etc. The question that we are investigating is that: with a finite budget, what is the best way to combine and allocate resources so as to maximize the performance level of the sensor network in terms of, for example, the target destruction rate and time?

In this paper, we propose an efficient algorithm to tackle this problem and show that it is effective in reducing the capturing time via simulations. With the data provided by the UGS network, the target vehicles' moving patterns are identified and they are further classified into wheeled and tracked categories of vehicles. The locations of the potential hostile vehicles are determined, predicted and tracked using different vehicle moving models, and the most efficient strategy for the deployment of the UAV fleet or other UGS clusters is decided, and eventually the UAV will confirm the targets and capture them. The rationale of our approach is that the low-cost low-capability sensors are strategically employed to cue and guide the high-performance but scare sensors to perform the task, through sensor management and strategic resource allocation and coordination placing the right sensors at the right locations on the right time.

The paper is organized as follows. Section 2 describes and formulates the problem at hand, and models this high dimensional design problem with a collection of autonomous decision-making entities, or agents. Section 3 discusses in details the collaborative signal and information processing algorithms for strategic resource allocation and coordination. Simulation results are given in Section 4 to evaluate the algorithms and demonstrate the benefits. The paper is then concluded.

\section{Problem Formulation and Modelling}

\section{Problem description and formulation}

To illustrate target detection and identification in complex environments with the aid of strategic resource allocation and coordination in heterogeneous sensor networks, we consider a specific problem where a sensor network consisting of UGS and UAVs is deployed on the battlefield, for the purpose of acquiring and eventually capturing the hostile vehicle targets. The vehicles are assumed to consist of three categories: hostile tracked vehicles, hostile wheeled vehicles, and civilian wheeled vehicles. In this scenario, the objective is to design rules for coordination and information sharing between UGS and UAVs to reduce the time required for clearing all the hostile vehicles with the constraint of total cost.

The system under consideration consists of three major components: a complex environment, vehicles, and sensors. The environment is a simplified two-dimensional urban grid of a pre-set, configurable size. The map is generated using a stochastic algorithm that ensures the resulting map is randomly created, but still follows a consistent pattern that resembles the layout of a city to a degree. Some buildings and forests are added into the map to complicate the terrain, which have impact on the sensors' accuracy such as the probability of detection and the probability of false alarm. Potential targets are roadbounded including hostile vehicles (either tracked or wheeled) and civilian vehicles (wheeled only). The vehicles traverse the environment by selecting starting points and destinations at random. They plan their routes by following the shortest path to their destinations, and are assumed to be confined in the environment if they are alive. The sensors consist of UGS and UAVs, which are imperfect, having a probability of reporting that a target is present when it isn't, as well as reporting that it is not present when it is. It is assumed that the local data fusion within a UGS node is available, and no fusion within neighboring nodes is provided locally, but All UGS can transmit the detections back to the base station in the command and control (C2) centre. UGS has the ability to detect a vehicle in the vicinity, and classify it into tracked or wheeled vehicles using for example the spectrum based method [3], and cannot accurately localize or track the target. Each UAV is equipped with cameras, the necessary image recognition facility, and the laser range finder, and would be able to identify and localize the targets if they are within the range and field of view (FOV) of the camera. Each UAV maintains a direct communication link to the base station.

\section{Agent-Based Modelling (ABM)}

We use a modeling approach known as agent-based modeling [29] to study and simulate the high dimensional design space problem at hand. In this approach, systems are represented as collections of autonomous decision-making entities, called agents. Each agent individually assesses its situation and makes decisions based upon a set of behavioral rules. At the simplest level, an agent-based model consists of a system of agents and the relationships between them. Even a simple agent-based model can exhibit complex behavioral patterns and provide valuable insight about the dynamics of the real-world applications that it emulates.

The benefits of ABM over other modelling techniques can be captured in three statements [30]: 1) ABM captures emergent phenomena; 2) $\mathrm{ABM}$ provides a natural description of the complex systems; 3) ABM is flexible. The ability of ABM to deal with emergent phenomena is the main driving force behind its success as a complex adaptive system modelling tool. The fact that it provides a natural framework to describe complex systems combined with its flexibility makes ABM the tool of choice for the problem at hand.

The parameters modelling the environment, sensors and vehicles, and the relationship and interaction between them are shown in Figure 1. The environment has impact on the vehicle behaviors, detection

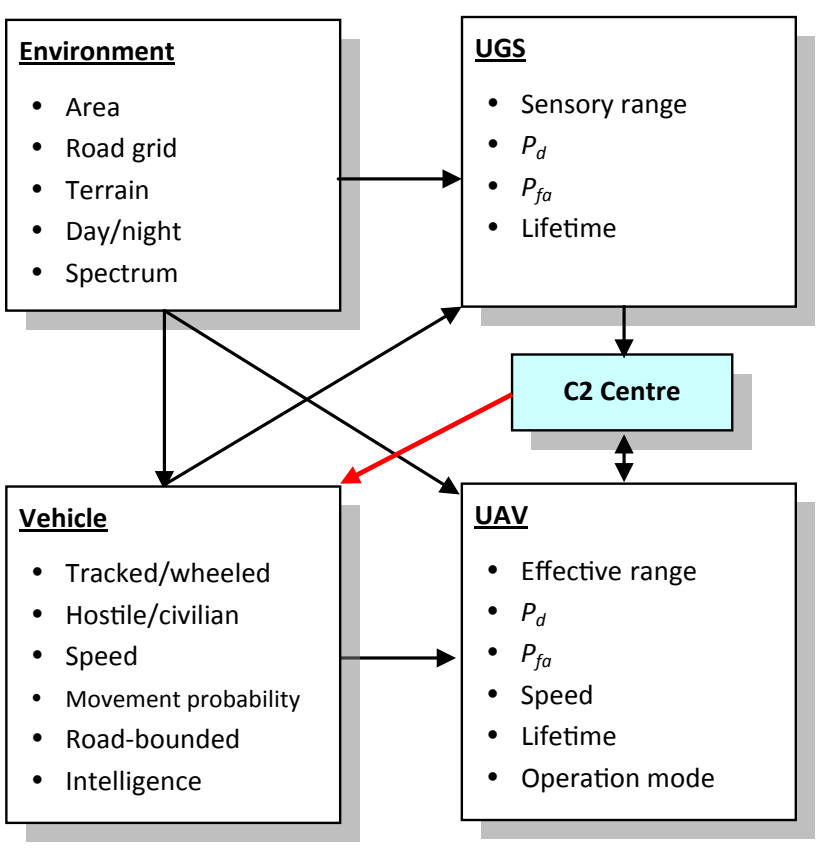

Figure 1: Agent-based system modelling. 
probability $P_{d}$ and false alarm probability $P_{f a}$ of UGS and UAVs due to the terrain and day/night variation. The $\mathrm{C} 2$ centre collects the rough target information from UGS, makes optimal task allocation and path scheduling for UAVs, and deploys munitions to destroy targets that are confirmed and localized by UAVs. Both UGS and UAVs have limited lifetime due to battery and petrol consumption, respectively.

\section{Solution Approach}

The algorithm for strategic resource allocation and coordination is shown below. Our approach specifies relatively simple local behavior to be adopted by each UGS and UAV, and the desirable global behavior arises from the interactions between them. N UGS are initially deployed at the junctions of roads to enhance the coverage efficiency because all the vehicles are assumed to be road bounded. If the vehicle behavior is known a priori, for example, the hostile vehicles are most likely coming from certain directions along certain roads, UGS can be placed strategically in those areas; otherwise, they are deployed randomly. At the beginning, M UAVs make autonomous search, where the term "autonomous" is compared to guided search cued by UGS. The UAVs may work in two ways: dividing the whole area into M zones and dispatching one UAV to each zone, or cooperatively searching for targets based on swarm intelligence [31]. Each UAV maintains a direct connection to the base station at the control centre, and all the UGS are networked and the detection information is sent to the base station either directly via the communication links or through multiple hops. The control centre maintains a two-dimensional map of the environment and the roads.

As illustrated in Figure 2, each UGS obtains its own sensor measurements, and makes a decision via the on-board data fusion. The results are contained in three categories: if no vehicles are detected in the vicinity, the result is 0 ; if tracked vehicles are detected, the result is 1 ; if only wheeled vehicles are detected, the result is 2 . Assume UGS

\section{The algorithm}

\author{
Initial placement of $N$ UGS \\ Autonomous search by $M U A V s$ \\ repeat \\ for each UGS do \\ Get own sensor measurements \\ Data fusion for target detection and classification \\ Send the decisions to $\mathrm{C} 2$ centre \\ end for \\ C2 centre: task allocation and path scheduling for UAVs \\ performing lower-priority tasks \\ for each UAV do \\ Receive commands from $\mathrm{C} 2$ center \\ Search the vicinity of destination UGS or make \\ autonomous search \\ if any targets are confirmed \\ Localize the target \\ Track the target while $\mathrm{C} 2$ center prepares \\ munitions to destroy it \\ Perform battle damage assessment (BDA) \\ end if \\ end for \\ until termination of operation
}

Figure 2: Algorithm for UGS and UAV coordination. function to be simple: it cannot count, accurately locate, or track the targets. This assumption helps to alleviate UGS' cost requirement. The results from the UGS are reported back to the control centre for data fusion which forms a preliminary search of the field. A probability method is then applied to the initial decisions to predict the vehicle locations to overcome the uncertainty caused by the delayed message, movement of the vehicles, and the inaccurate local information. A major challenge is how to associate the UGS detections with the correct targets, a method based on the principle of machine learning [32] is utilized in this work. After predicting the vehicle locations, the control centre designs the capture strategies. There are three options: 1) plan the most efficient pursuit trajectories of the UAV fleet based on the swarm intelligence; 2) rapidly deploy another set of UGS (with artillery or by operators) in the direction of the vehicle movement to track the vehicles; and 3) combined actions of decisions 1) and 2).

Assume $n$ UGS report tracked detection 1, $n$ UGS report wheeled detection 2, and the other $N-n_{t}-n_{w}$ UGS report null detection 0 . If, $n_{t}+n_{w} \geq M$ all M UAVs are involved in the task allocation and coordination; otherwise only $n_{t}+n_{w}$ UAVs are involved and the remaining UAVs make autonomous search. Strategic deployment of multiple UAVs means task allocation and path planning for some destinations that must be visited and some risk sites that must be avoided. The flying time is a criterion used to measure the effectiveness of a schedule. A good schedule can guide the UAVs to all destination UGS as quickly as possible, before the targets move far away. When only one UAV is considered, the problem is similar to traveling salesman problem (TSP). In the scenarios involving multiple UAVs, the problem resembles Job-Shop Scheduling Problem (JSSP) [33]. Both TSP and JSSP are NP-hard and intensively studied in literature. In our system, the Particle Swarm Optimization (PSO) algorithm [34] is used to allocate tasks and design paths for each UAV. PSO is a recent addition to evolutionary algorithms first introduced by Eberhart and Kennedy in 1995. The foundation of PSO is based on the hypothesis that social sharing of information among conspecifics offers an evolutionary advantage. Partially inspired by animal social behaviors such as flocking of birds, PSO originally intends to graphically mimic the graceful way in which they find their food sources and save themselves from predators. It should be noted that if $n_{t}+n_{w}>>M$, it is not necessary to devise a complete schedule to visit all $n_{t}+n_{w}$ UGS, since after a long time, the targets have moved far away. The number of destination UGS considered in our algorithm is determined by the amount of UAVs, the sensory ranges of UGS and UAVs, the speed of the target vehicles and UAVs, target behavior, and etc. Another rule that should be pointed out is that, the task of searching for tracked vehicles has higher priority than that for wheeled vehicles, which has higher priority than autonomous search. Based on this rule, UAVs can be interrupted from current task and reallocated to perform higher priority tasks.

The algorithm will not terminate automatically. Even if all hostile vehicles have been destroyed (but this information has not been confirmed and notified to the control centre), civilian vehicles continue triggering UGS' detections and UAVs' guided search.

\section{Simulation Results}

The design space for the problem of resource allocation and behavioral coordination is complicated and high-dimensional, involving the amount of sensors, detection range and accuracy of sensors, coordination rules between sensors, in addition to the environment characteristics and target behaviors. In this section, we demonstrate 1) the effectiveness of agent-based modelling to this 
type of problem, 2) sensor selection and coordination strategies at the constraint of limited budget, and 3) benefits introduced by strategic sensor management and coordination. For the sake of simplicity, only two key dimensions, the amount and capability of sensors are explored, but we stress that other dimensions can be similarly treated.

We consider an urban area of $40 \mathrm{~km}$ by $40 \mathrm{~km}$ including 2500 uniformly distributed junctions. The target vehicles consist of 40 hostile tracked vehicles, 40 hostile wheeled vehicles and 400 civilian wheeled vehicles. All the vehicles are road bounded with a movement probability of $50 \%$, and at a speed between $40 \mathrm{~km} / \mathrm{h}$ and $80 \mathrm{~km} / \mathrm{h}$. UAV has two operation modes: cruising mode at a speed of $200 \mathrm{~km} / \mathrm{h}$, and searching mode at a speed of $100 \mathrm{~km} / \mathrm{h}$. The results are calculated based on an average over 30 runs.

In the first experiment, the amount of required UGS and UAVs is studied. Assume UGS' sensory range $\mathrm{r}=0.5 \mathrm{~km}$, the probability of detection $P_{d}=0.8$, and the probability of false alarm $P_{f a}=0.15$; UAV's sensory range $r=3 \mathrm{~km}, P_{d}=0.8, P_{f a}=0.05$. Figure 3 shows the effectiveness, in terms of the half target destruction time, versus the number of UAVs, with certain amount of UGS. It is evident that, in all cases we have a consistent reduction in the target destruction time with the increase of UAVs, which corresponds to a higher cost. It is interesting to note that when the number of UAVs is greater than 5, too many UGS (for example, when the number of UGS increases from 40 to 100 ) may cause drawbacks, since $P_{f a}$ is virtually increased due to large civilian vehicle detections which then triggers more vain UAV search. Another interesting point is the nonlinear change nature of the curves, which is common in the complex systems. For example, we can tell that when the UGS number is 40 , increasing UAVs from 3 to 4 has a much greater impact than, say increasing UAVs from 9 to 10 . The ability to find "tipping points" is a key driver of success in the analysis of complex systems of this type.

In the second experiment, the impact of UGS' detection range $r$, $P_{d}$ and $P_{f a}$ on the system effectiveness is studied. Figure 4 shows the half target destruction time versus $r$ with certain $P_{d}$ value and $P_{f a}=0.15$. Although the curves are not strictly monotonic due to the average over small number of trials, the trends and impact of these parameters on the target destruction time are clearly demonstrated. In general,

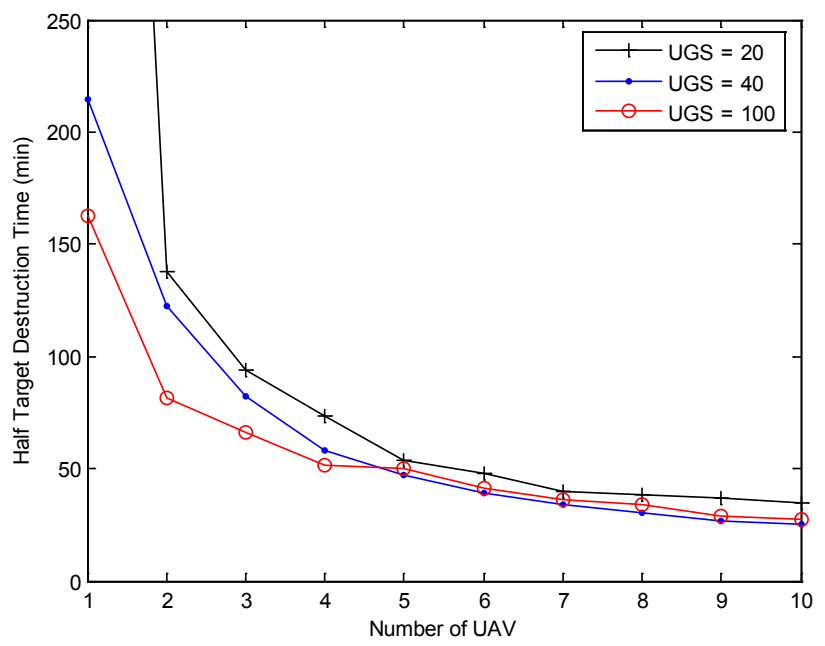

Figure 3: Impact of sensor number on system performance.

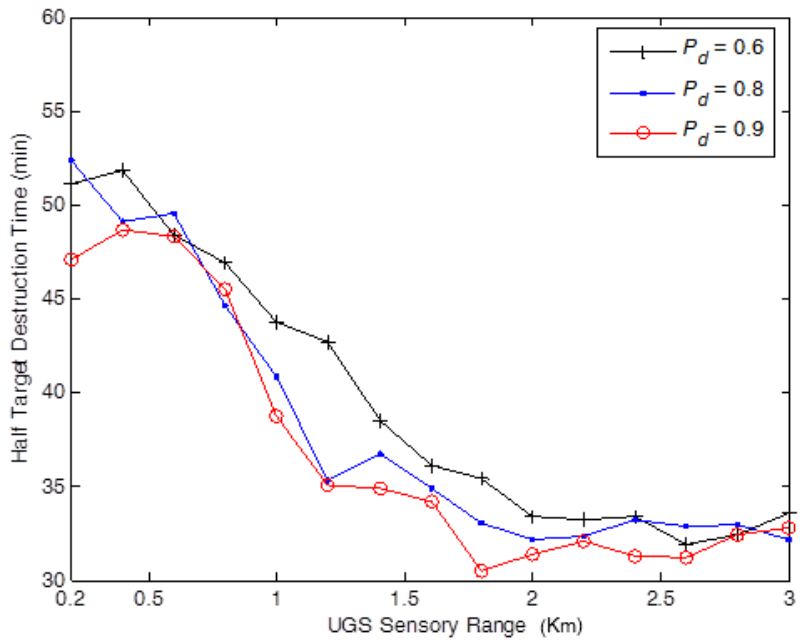

Figure 4: Impact of sensory range and accuracy on system performance.

larger detection range and higher $P_{d}$ of UGS, which corresponds to a higher cost, lead to faster target destruction. Figure 5 shows the target destruction time versus $P_{d}$ with certain $P_{f a}$ and $r=1 \mathrm{~km}$. It is interesting to note that when $P_{d}$ is high (for example, above 0.8 ), the system is more sensitive to $P_{f a}$ by showing large fluctuations in the curves with $P_{d}$ changing; however, when $P_{d}$ is moderate (for example, between 0.6 and 0.8 ), the performance is more dominated by $P_{d}$, and lower $P_{f a}$ causes more fluctuations as $P_{d}$ varies. This phenomenon is of great practical interests to the system designers. Again, tipping points are identified in the system.

In the third experiment, the proposed method of UGS-guided search is compared with UAV's autonomous search. The UGS settings are $r=1, P_{d}=0.8, P_{f a}=0.2$. As shown in Figure 6, the solution involving 4 UAVs and 40 UGS is much more effective in terms of the half target destruction time, especially when the number of remaining targets is moderate or small. UAV is expensive high-end equipment, so the solution of 4 UAVs and a set of UGS is much cheaper than that involving 8 UAVs. For guided search, tracked vehicles are diminished faster than wheeled vehicles, since higher priority attention is given to tracked detections in our system based on our rules, and due to the assumption that all civilian vehicles are wheeled vehicles. For autonomous search, two types of vehicles are destroyed with a similar rate.

\section{Conclusions and Future Work}

In this paper, an approach for target detection and acquisition with a heterogeneous sensor network through sensor management and strategic resource allocation and coordination is presented. We focus on the problem of designing a network system in which resource constraints (such as the limited budget) and system behavior and performance must be addressed simultaneously. Although our work is only embryonic in nature, the results already offer significant insight into the resource selection and network operation, and demonstrate the great benefits in a sample battlefield application.

As future work, we will explore the applications of evolutionary algorithms as techniques to search the high dimensional problem space for an optimal solution, in terms of the amount of each type of sensors, the sensor range and accuracy, the inter-node data fusion algorithms, 
Citation: Li M, Lu Y, He B (2013) Collaborative Signal and Information Processing for Target Detection with Heterogeneous Sensor Networks. Sensor Netw Data Commun 1: 112. doi: 10.4172/2090-4886.1000112

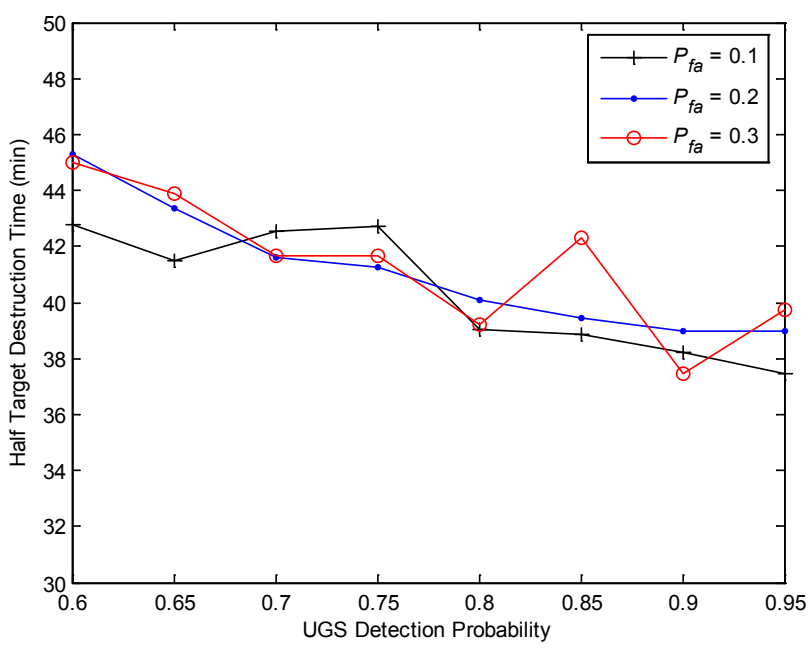

Figure 5: Impact of sensor accuracy on system performance.

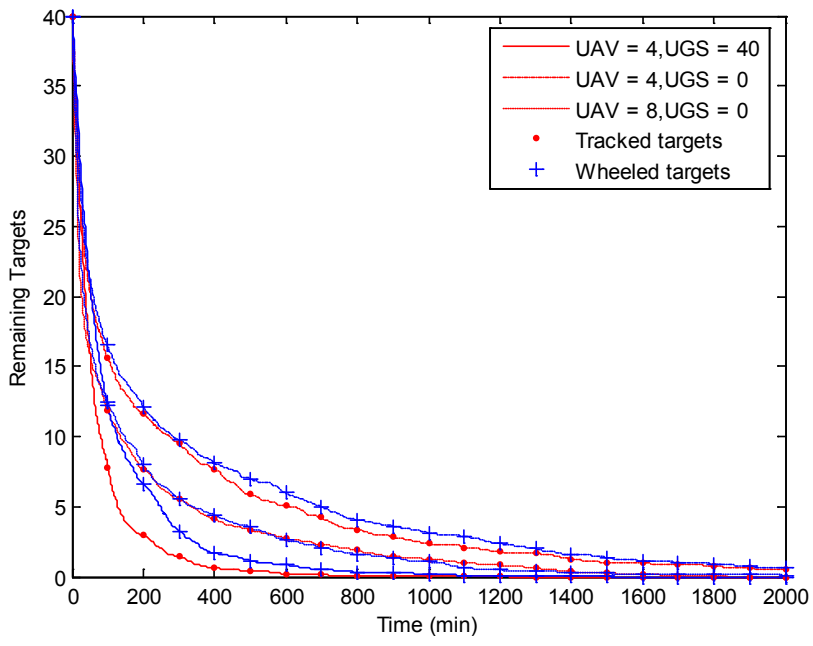

Figure 6: Comparison between UGS-guided search and autonomous search

network level resource allocation rules, with multiple constraints such as budget, communication range and link failure, and etc.

\section{References}

1. Culler D, Estrin D, Srivastava M (2004) Overview of sensor networks. IEEE Computer 37: 41-49.

2. Sheng $\mathrm{X}, \mathrm{Hu} Y$ (2005) Maximum likelihood multiple-source localization using acoustic energy measurements with wireless sensor networks. IEEE Transactions on Signal Processing 53: 44-53.

3. Li D, Wong K, Hu Y, Sayeed AM (2002) Detection classification and tracking of targets. IEEE Signal Processing Magazine 19: 17-29.

4. Reid DB (1979) An algorithm for tracking multiple targets. IEEE Transactions on Automatic Control 24: 843-854

5. Oh S, Russell S, Sastry S (2004) Markov chain Monte Carlo data association for general multiple-target tracking problems. Proceedings of the 43rd IEEE Conference on Decision and Control Bahamas.

6. Li MH, Lu YL (2004) Improving the performance of GA-ML DOA estimator with a resampling scheme. Signal Processing 84: 1813-1822.

7. Li MH, Lu YL (2006) Dimension reduction for array processing with robus interference cancellation. IEEE Transactions on Aerospace and Electronic Systems 42: 103-112.
8. Li MH, Lu YL (2005) Null-steering beam space transformation design for robust data reduction. Proceedings of 13th European Signal Processing Conference.

9. Li MH, Lu YL (2009) Source bearing and steering-vector estimation using partially calibrated arrays. IEEE Transactions on Aerospace and Electronic Systems 45: 1361-1372.

10. Li MH, Lu YL (2008) Maximum likelihood DOA estimation in unknown colored noise fields. IEEE Transactions on Aerospace and Electronic Systems 44 1079-1090.

11. Li MH, Lu YL (2002) Genetic algorithm based maximum likelihood DOA estimation. Proceedings of Radar Conference 502-506.

12. Li K, Lu YL, Li MH (2005) Approximate formulas for lateral electromagnetic pulses from a horizontal electric dipole on the surface of one-dimensionally anisotropic medium. IEEE Transactions on Antennas and Propagation 53: 933-937.

13. He B, Liang Y, Feng X, Nian R, Yan T, et al (2012) AUV SLAM and experiments using a mechanical scanning forward-looking sonar. Sensors 12: 9386-9410.

14. Li MH, McGuire M, Ho KS, Hayward G (2010) Array element failure correction for robust ultrasound beamforming and imaging. Proceedings of 2010 IEEE International Ultrasonics Symposium.

15. Li MH, Ho KS, Hayward G (2009) Beamspace transformation for data reduction using genetic algorithms. Proceedings of IEEE International Ultrasonics Symposium.

16. Li MH, Lu YL (2007) A refined genetic algorithm for accurate and reliable DOA estimation with a sensor array. Wireless Personal Communications 43: 533-547.

17. Li MH, Lu YL (2008) Angle-of-arrival estimation for localization and communication in wireless networks. Proceedings of 16th European Signal Processing Conference.

18. Li MH, Lu YL, Chen HH, Wang B, Chen IM (2009) Angle of arrival (AOA) estimation in wireless networks. Invited chapter Wireless networks: Research technology and applications Nova Science Publishers Inc. New York. 135-164.

19. Wang B, Li MH, Lim HB, Ma D, Fu C (2009) Energy efficient information processing in wireless sensor networks. Guide to Wireless Sensor Networks London Springer. Chapter 1: 1-26.

20. Li MH, Lu YL, Wee L (2006) Target detection and identification with a heterogeneous sensor network by strategic resource allocation and coordination. Proceedings of 6 th International Conference on Intelligent Transportation Systems Telecommunications 992-995.

21. Li MH, Ho KS, Hayward G (2010) Accurate angle-of-arrival measurement using particle swarm optimization. Wireless Sensor Network 2: 358-364.

22. Li MH, Wang B, Lu YL, Zhou MT, Chen IM (2010) Smart antenna in intelligent transportation systems. Wireless Technologies in Intelligent Transportation Systems Nova Science Publishers Inc. New York. Chapter 3: 51-84.

23. Li MH, Lu YL (2007) Optimal direction finding in unknown noise environments using antenna arrays in wireless sensor networks. Proceedings of $7^{\text {th }}$ International Conference on Intelligent Transportation Systems Telecommunications 332-337.

24. Li MH, Lu YL (2007) Maximum likelihood processing for arrays with partially unknown sensor gains and phases. Proceedings of 7 th International Conference on Intelligent Transportation Systems Telecommunications 185-190.

25. Gongzhang R, Li MH, Lardner T, Gachagan A, Hayward G (2012) Robust defect detection in ultrasonic nondestructive evaluation (NDE) of difficult materials. Proceedings of 2012 IEEE International Ultrasonics Symposium.

26. Lardner T, Li MH, Gongzhang R, Gachagan A (2012) A new speckle noise suppression technique using cross-correlation of array sub-apertures in ultrasonic NDE of coarse grain materials. Proceedings of Review of Progress in Quantitative Nondestructive Evaluation (QNDE).

27. Li MH, Hayward G, He B (2011) Adaptive array processing for ultrasonic nondestructive evaluation. Proceedings of 2011 IEEE International Ultrasonics Symposium.

28. Li MH, Hayward G (2012) Ultrasound nondestructive evaluation (NDE) imaging with transducer arrays and adaptive processing. Sensors 12: 42-54

29. Bonabeau E (2002) Agent-based modeling: Methods and techniques for simulating human systems. Proceedings National Academy of Science 99 7280-7287. 
Citation: Li M, Lu Y, He B (2013) Collaborative Signal and Information Processing for Target Detection with Heterogeneous Sensor Networks. Sensor Netw Data Commun 1: 112. doi: 10.4172/2090-4886.1000112

Page 6 of 6

30. Parunak HV, Savit R, Riolo RL (1998) Agent-Based Modeling vs. EquationBased Modeling: A case study and users' guide. Proceedings of Multi-agent systems and Agent-based Simulation (MABS'98): 10-25.

31. Kadrovach BA, Lamont GB (2002) A particle swarm model for swarm-based networked sensor systems. Proceedings of ACM symposium on Applied computing: 918-924

32. Vercauteren T, Guo D, Wang X (2004) Joint multiple target tracking and classification in collaborative sensor networks. Proceedings of IEEE International Symposium on Information Theory (ISIT).

33. Fang HL, Ross P, Corne D (1993) A promising genetic algorithm approach to job-shop scheduling rescheduling and open-shop scheduling problems. Proceedings of the 5th International Conference on Genetic Algorithms: 375-382.

34. Eberhart RC, Kennedy J (1995) A new optimizer using particle swarm theory. Proceedings of the 6th Symposium on Micro Machine and Human Science, Nagoya, Japan:39-43. 